\title{
SMART PHARMACY MONITORING SYSTEM BASED ON MQTT PROTOCOL USING RFID AND RASPBERRY PI
}

\author{
Fahad Ghalib Abdulkadhim \\ University of Kufa \\ Faculty of Computer science and math \\ Najaf, Iraq \\ fahadg.abdulkadhim@uokufa.edu.iq
}

Zhang $\mathbf{Y i}^{1}$

Mudassar Khalid

${ }^{1}$ School of electronic and information engineering

North-western Polytechnical University

127 West Youyi Road, Beilin District, Xi'an Shaanxi, 710072, P.R.China

\begin{abstract}
Smart mobile pharmacy has become a necessary requirement in improving the quality of healthy life in the last five years. Determining the type of medication and calculating the dose for each patient according to the patient's condition, monitoring the patient at the post and how to take the doses on time have a great effect on improving the patient's condition as soon as possible.

In this paper, a system of monitoring, and guidance based on potency management have been designed to contain a cloud data storage platform and its implementation to solve the above problem.

The system is achieved by connecting each item (Medicine bottle) to a device (RFID) to verify that the patient has arrived in the bottle the correct medicine and is equipped with suppression of the treatment of the drug contains the sensors of weight to be sent all information to the control unit to be over the Internet through the routing portal.

All data is stored in the first two data folders, which are static and the second is mobile. Synchronization between the two stations is performed to update the information and will be executed on a server that uses an MQTT protocol to register and integrate messages on the platform. The results of the experiment show the speed of guidance and reduce cost and reduce energy.
\end{abstract}

Keywords: MQTT, RFID, internet of things, Medicine bottle, sensors, raspberry Pi.

DOI: $10.21303 / 2461-4262.2020 .001207$

\section{Introduction}

In this paper the authors describe the designing/developing of a system that can be applied to any object or environment with little or no modifications and easily used by any person. The system will provide full remote and secure control and monitoring of sensor networks, via an online platform, that can be applied to any non-smart object or environment allowing them to be connected to the Internet and to the user. With the possibility to add a set of rules and Artificial Intelligence it is possible to improve the efficiency leading to potential gains, such as energy and low cost. Although the main goal is to create a low-cost system that is flexible in the sense that it can be easily adapted to any specification, in this paper the practical functionality will be tested and evaluated in a pharmacy smart application, with an application capable of monitoring the patient.

The mobile pharmacy facilitates the practice of clinical pharmacy and patient monitoring of mobile devices. Electronic pharmacy or e-health has generally emerged as a subset of health in the form of the use of ICT, satellites, computers, smartphones, and so forth.

In this paper, emphasis has been placed on a monitoring, monitoring, extension and telemedicine system as a type of mobile health application, including the use of mobile devices and sensors in collecting health information, providing health care information and advice to practitioners, researchers and patients in real time and monitoring how to use a temporary identification appropriate doses and doses to improve the effectiveness of treatment [1].

Smart Pharmacy Monitoring System is divided into two parts, the first section is the hardware and contains the hardware devices used in the system that deals with the physical stopper, and the second part, which deals with software that manages the system in all its details and is within the application layer, the network topology of the system is shown as Fig. 1. 